This is an accepted manuscript, The final version can be found in Advanced Engineering Materials DOI:10.1002/adem.201400565

DOI: 10.1002/adem.201400565 ((please add manuscript number))

\title{
Enhanced Synthesis of Metal-Organic Frameworks on the Surface of Electrospun Cellulose Nanofibers**
}

By Johannes Thunberg*, Elina Laurila, Stephen P. Argent, Neil R. Champness, Savannah Zacharias, Gunnar Westman* and Lars Öhrström

[*] Johannes Thunberg, and Gunnar Westman

Wallenberg Wood Science Center and Department of Chemical and Biological Engineering, Chalmers University of Technology

Kemigarden 4, Gothenburg, SE-41296, Sweden

E-mail: johannes.thunberg@chalmers.se; westman@chalmers.se

Elina Laurila and Lars Öhrström

Department of Chemical and Biological Engineering, Chalmers University of Technology Kemigarden 4, Gothenburg, SE-41296, Sweden

Stephen P. Argent, Neil R. Champness

School of Chemistry, University of Nottingham, University Park, Nottingham, NG7 2RD, UK

Savannah Zacharias

Department of Chemistry, University of Cape Town, Rondebosch 7700, South Africa

[**] JT and GW gratefully acknowledge the Knut and Alice Wallenberg Foundation for financial support through the Wallenberg Wood Science Center. NRC acknowledges the receipt of a Royal Society Wolfson Merit Award. LÖ, and EL thank the Swedish Research Council for support and SZ also for a Swedish Research Links grant.

((Supporting Information is available online from Wiley InterScience or from the author))

This study reports the in situ crystal growth of HKUST-1 on electrospun cellulose nanofibers.

Two different methods for introducing carboxyl groups on the nanofiber surface were used,

HKUST-1 was then synthesized on the cellulose nanofiber surface using a layer-by-layer

approach. The distribution of HKUST-1 on the nanofiber surface was highly dependent on the type of anionic pretreatment. The loading of HKUST-1 on the nanofiber surface could be controlled by the layer-by-layer synthesis and the BET surface area could be increased by a factor of 44 to $440 \mathrm{~m}^{2} / \mathrm{g}$.

Metal-organic framework (MOF) materials are 3D network structures consisting of a metallic unit and organic ligands, and are usually synthesized via self-assembly. MOFs have versatile 
This is an accepted manuscript, The final version can be found in Advanced Engineering Materials DOI:10.1002/adem.201400565

properties that are important in optical devices, imaging and magnetic materials. ${ }^{[1]}$. The specific porosity of MOF materials have been the focus of interest for many researchers due to possible application in gas storage, catalysis and separation. ${ }^{[2]}$. MOFs are usually prepared as powder bulk materials but in many applications such as sensors, membranes and catalytic coatings MOFs need to be immobilized as thin films, layers or coatings in order to be useful. $^{[3]}$ This realization has been the driving force for research focusing on materials incorporating MOFs. The strategy is often to maintain the mechanical stability of the substrate material and then incorporate MOFs, which can act as a functional chemical modification. Several methods have been used to prepare MOFs films, including direct growth of films during synthesis, evaporation reaction mixture and layer-by-layer methods. ${ }^{[3-5]}$ MOFs can be synthesized directly on the surface of the substrate material, but often the surface is modified in order to make the anchoring of MOFs easier. Surface modification has focused on making the surface anionic, consequently giving the cationic metal anchor points. ${ }^{[3],[4],[6]}$ Previously, MOFs have been synthesized on natural fiber materials such as silk, cotton and pulp fibers. ${ }^{[7]}$ Recently, researchers have started to create nanofiber/MOF materials using electrospun polymeric fibers. Ostermann et al. ${ }^{[8]}$ published a method for incorporating zeolitic imidazolate framework (ZIF-8) crystals into nanofibers during the electrospinning process. Other researchers have also made materials with MOF crystals embedded within electrospun nanofibers. ${ }^{[9],[10]}$ However, this approach decreased the adsorption properties of the MOF due to the surrounding polymer acting as a diffusion barrier. ${ }^{[8]}$ Another approach has been to utilize the large specific surface area of electrospun nanofibers. Centrone et al. ${ }^{[11]}$ tried to synthesize MOF materials directly on the surface of electrospun polyacrylonitrile nanofibers using microwave irradiation, but the nanofibers melted together and lost their structure during microwave irradiation. Very recently, Jin et al. ${ }^{[12]}$ published a method for synthesizing ZIF-8 on the surface of electrospun polyimide nanofibers. During the course of our work Lian et al. ${ }^{[13]}$ published a paper describing ZIF- 8 synthesis on the surface of electrospun polyurethane 
This is an accepted manuscript, The final version can be found in Advanced Engineering Materials DOI:10.1002/adem.201400565

nanofibers using chromium trioxide to oxidize the nanofiber surface as a pretreatment. A more sustainable approach to a nanofiber/MOF material would be to use an easily oxidized and natural polymer that can be electrospun. Cellulose is the most abundant organic polymer on Earth and a sustainable raw material. ${ }^{[14]}$ Native cellulose can be electrospun directly from ionic liquids. ${ }^{[15]}$ However, electrospinning of the derivative cellulose acetate is more efficient because a high concentration of polymer can be used in the spinning solution. Subsequent removal of acetyl groups using alkaline hydrolysis regenerates the cellulose. ${ }^{[16]}$ Since cellulose is a linear polymer of $\beta(1 \rightarrow 4)$-D-glucopyranose units, it has no natural anionic anchor point for the crystal growth of a MOF.

In this work we evaluated MOF growth and distribution on the surface of pretreated anionic electrospun cellulose. Two established methods for introducing anionic carboxyl groups on the cellulosic surface were used, carboxymethylation using sodium chloroacetate and adsorption of carboxymethyl cellulose (CMC). ${ }^{[17]}$ The MOF HKUST- ${ }^{[18]}$, also known as CuBTC, was subsequently synthesized on the pretreated cellulose nanofibers using a layer-bylayer approach. In this approach the copper metal was anchored to the anionic groups and then ligands were introduced, which could coordinate to the anchored metals (Scheme 1).

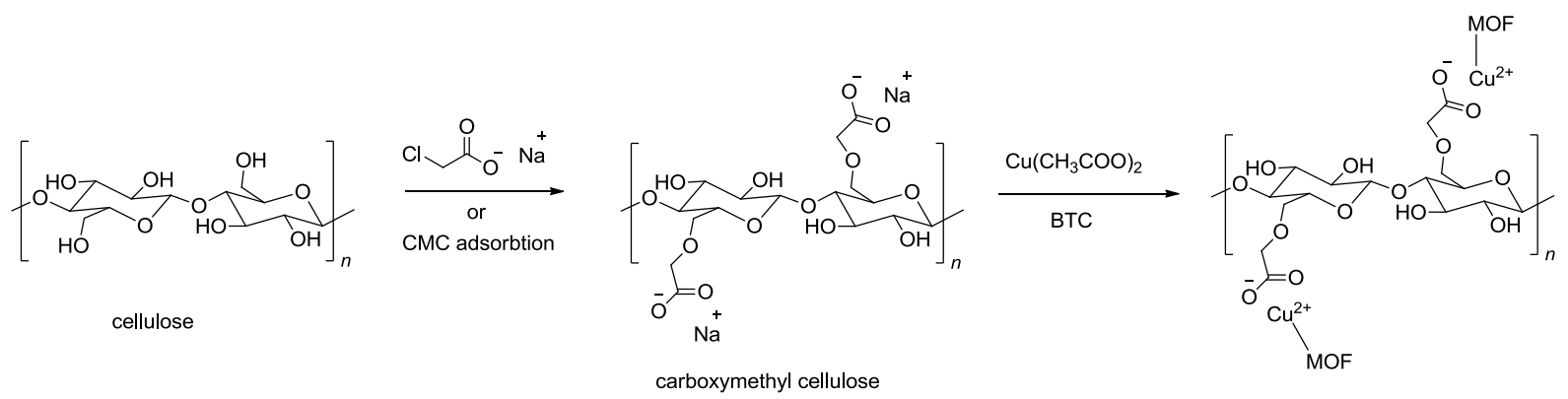

Scheme 1. Scheme for synthesis of HKUST-1 on carboxymethylated and CMC adsorbed cellulose nanofibers

Two sets of samples were prepared using either 8 or 32 synthesis cycles. After HKUST-1 synthesis, the electrospun nanofibers of unmodified cellulose and CMC adsorbed cellulose displayed inhomogeneous coloring, while the carboxymethylated cellulose nanofibers were 
This is an accepted manuscript, The final version can be found in Advanced Engineering Materials DOI:10.1002/adem.201400565

homogeneously cyan/green colored (Figure S1, Supporting Information). This observation together with the increase in HKUST-1 content (Table 1) indicates that, as anticipated, the anionic surface treatment enhances HKUST-1 crystal growth on the nanofibers surface.

Table 1. HKUST-1 content of cellulosic nanofiber samples treated with layer-by-layer synthesis method.

\begin{tabular}{lll} 
& \multicolumn{2}{l}{ HKUST-1 content ${ }^{[\mathrm{a}]}$} \\
\cline { 2 - 3 } Nanofiber type & 8 cycles & $\begin{array}{l}32 \\
\text { cycles }\end{array}$ \\
\hline Cellulose & $9.8 \%$ & $32.2 \%$ \\
CMC adsorbed & $9.2 \%$ & $38.9 \%$ \\
Carboxymethylated & $11.8 \%$ & $38.7 \%$ \\
\hline
\end{tabular}

[a] HKUST-1 content was estimated from TGA ash content, details are available in Supporting Information

There were no signs of HKUST-1 delaminating from the nanofibers during washing, drying or handling of the materials after HKUST-1 synthesis, which indicate that HKUST-1 was immobilized in the nanofibers surface. The presence of HKUST-1 in the nanofibers materials was verified with X-ray diffraction (XRD) and FTIR. The XRD patterns of all the HKUST-1 functionalized cellulose nanofibers are shown in Figure 1, together with the XRD pattern of pure HKUST-1 and a HKUST-1 reference pattern $\left(\right.$ CSD-DIHVIB ${ }^{[19]}$ ) generated by the software Mercury 3.3. 
This is an accepted manuscript, The final version can be found in Advanced Engineering Materials DOI:10.1002/adem.201400565

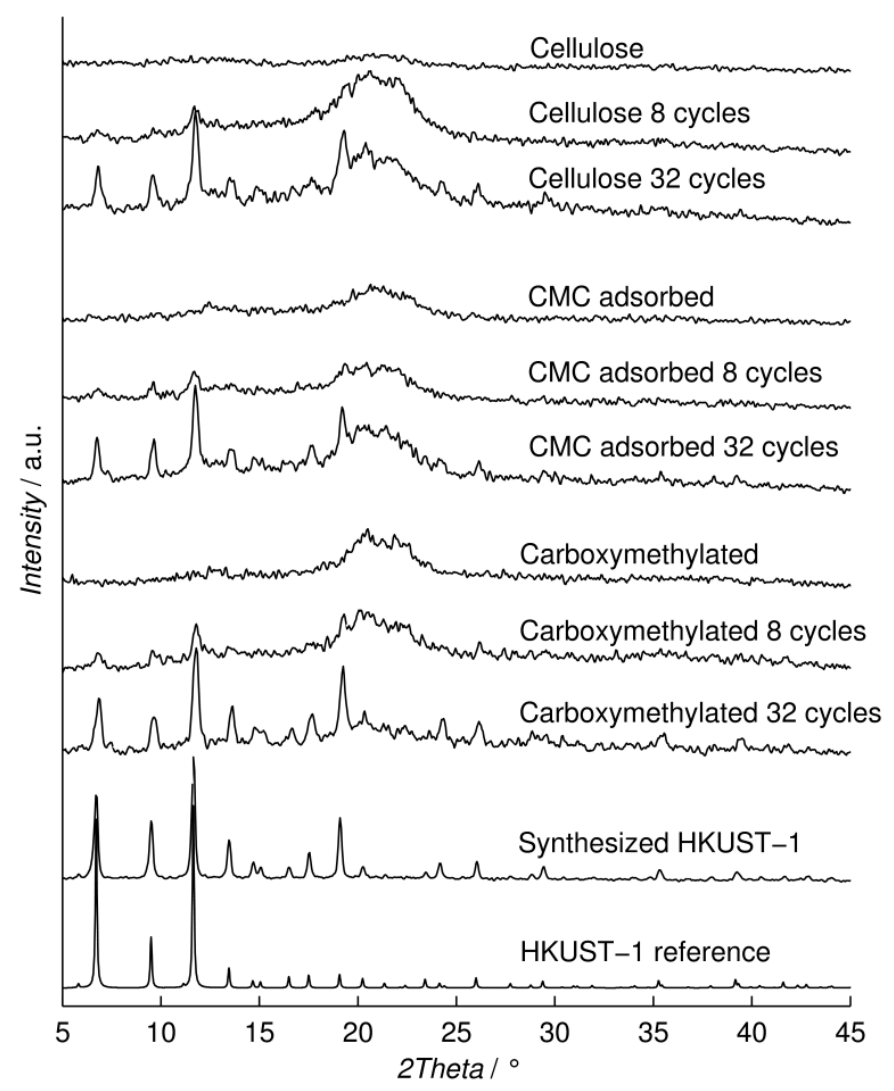

Figure 1. Combined XRD patterns of cellulose, CMC adsorbed cellulose and carboxymethylated cellulose. All nanofibers materials were functionalized with HKUST-1 using 8 or 32 synthesis cycles. Also shown are XRD pattern of pure HKUST-1 and a HKUST1 reference pattern.

The high similarity of these patterns proves the formation of HKUST-1 crystallites in the all cellulosic nanofiber materials. The general trend in the XRD patterns is an increased intensity of the characteristic HKUST-1 peaks as the number of synthesis cycles increase. In addition, FTIR spectrums of all the HKUST-1 functionalized nanofibers show strong characteristic vibration bands at $729 \mathrm{~cm}^{-1}, 760 \mathrm{~cm}^{-1}, 1373 \mathrm{~cm}^{-1}, 1447 \mathrm{~cm}^{-1}$ and $1637 \mathrm{~cm}^{-1}$ (Figure S2, Supporting Information). These vibration bands were in good agreement with published data on HKUST-1. ${ }^{[20]}$ The broad vibration band at $1200-900 \mathrm{~cm}^{-1}$ that stems from the cellulose nanofibers seem to decrease in intensity as the number of synthesis cycles increase, which indicate that both HKUST-1 and cellulose is present in the nanofiber materials. 
This is an accepted manuscript, The final version can be found in Advanced Engineering Materials DOI:10.1002/adem.201400565

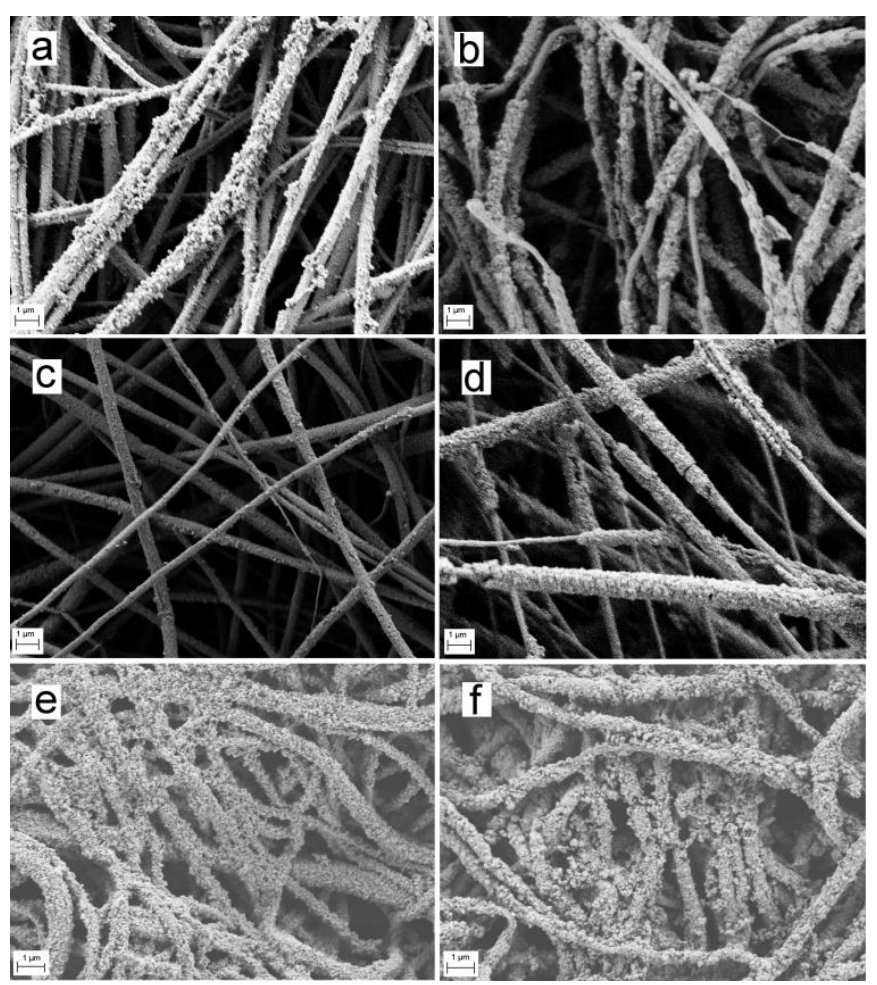

Figure 2. SEM images of (a) cellulose nanofibers 8 synthesis cycles, $(b)$ cellulose nanofibers 32 synthesis cycles, (c) CMC adsorbed nanofibers 8 synthesis cycles, (d) CMC adsorbed nanofibers 32 cycles, (e) carboxymethylated nanofibers 8 cycles, $(f)$ carboxymethylated nanofibers 32 cycles. Scale bars are $1 \mu \mathrm{m}$.

Scanning Electron Microscopy (SEM) images of the electrospun cellulose nanofibers revealed that their diameter ranged between $300 \mathrm{~nm}$ and $1500 \mathrm{~nm}$. Furthermore, HKUST-1 particles are visible on the surface of non pretreated cellulose nanofibers after 8 cycles (Figure 2, a) and after 32 cycles the nanofibers appear to be unevenly covered by HKUST-1 (Figure 2, b). Partly covered nanofibers are also visible in the CMC adsorbed nanofibers (Figure 2, d), which indicate adhesion of HKUST-1 to CMC treated nanofibers was low. However, it is also possible that the HKUST-1 exhibited uneven crystal growth due to fewer carboxyl anchor points. The HKUST-1 functionalized carboxymethylated nanofibers (Figure 2, e and f) display a uniform growth of HKUST-1 over the whole nanofiber surface, both in the 8 cycle sample and the 32 cycle sample. This observation in the carboxymethylated nanofibers indicate that carboxyl anchor points are evenly distributed on the nanofibers surface and that adhesion between the nanofibers and HKUST-1 is high. $\mathrm{N}_{2}$ adsorption measurements were made in order to confirm the gas adsorption properties of the HKUST-1 functionalized 
This is an accepted manuscript, The final version can be found in Advanced Engineering Materials DOI:10.1002/adem.201400565

cellulose nanofibers. Adsorption measurements were performed on a sample of carboxymethylated cellulose nanofibers with 32 HKUST-1 synthesis cycles. This material was used since it had high HKUST-1 content that was evenly distributed on the nanofiber surface. Adsorption and desorption data (Figure S3, Supporting Information) show that $\mathrm{N}_{2}$ adsorption dramatically increase for the HKUST-1 functionalized cellulose nanofibers compared to unmodified cellulose nanofibers. The overall gas adsorption for the unmodified cellulose was low, indicating that the sample had very low porosity and that the porosity observed in HKUST-1 functionalized cellulose nanofibers was a result of the MOF material. The BET surface area was $440 \mathrm{~m}^{2} / \mathrm{g}$ for the HKUST-1 functionalized cellulose nanofibers, while only $10 \mathrm{~m}^{2} / \mathrm{g}$ for the unmodified cellulose nanofibers.

In summary, HKUST-1 was synthesized on pretreated anionic electrospun cellulose which gave the resulting MOF/cellulose material a nano-porous structure suitable for gas adsorption. The anionic pretreatment enhanced the synthesis of HKUST-1 on the nanofibers surface. The carboxymethylated electrospun cellulose was best suited for HKUST-1 functionalization, since the HKUST-1 crystals became evenly distributed on the nanofiber surface. The approach of surface modification of cellulose for the synthesis of MOF/cellulose materials presented in this work opens up possibilities for MOF synthesis on other cellulosic materials, such as nano crystalline cellulose and micro fibrillated cellulose.

\section{Experimental}

\section{Electrospinning}

$18 \%$ wt. cellulose acetate (acetyl content of $39.8 \% w t$ and $M_{n}$ of 30,000 ) was dissolved in dimethylacetamide/acetone $(11: 14 \mathrm{v} / \mathrm{v})$. The cellulose acetate solution electrospun at $20^{\circ} \mathrm{C}$ and relative humidity of $65 \%$ for $3 \mathrm{~h}$ with a feed rate of $0.350 \mathrm{~mL} \mathrm{~h}^{-1}$ and an applied voltage of $18-20 \mathrm{kV}$. The electrospinning equipment consisted of a high voltage power supply, a NE- 
This is an accepted manuscript, The final version can be found in Advanced Engineering Materials DOI:10.1002/adem.201400565

1000 syringe pump and a $10 \mathrm{ml}$ syringe connected to a blunt-nozzle stainless steel needle. For collection of the nanofiber mats a $2.5 \mathrm{~cm}$ wide cylindrical $(10 \mathrm{~cm}$ diameter $)$ grounded collector rotating at $25 \mathrm{rpm}$ was used. Collector to needle distance was $15 \mathrm{~cm}$. The cellulose acetate nanofiber mats were dried at $80^{\circ} \mathrm{C}$ over night and then immersed in $\mathrm{NaOH}(0.1 \mathrm{~mol}$ $\left.\mathrm{dm}^{-3}\right)$ in ethanol:water $(1: 1 \mathrm{v} / \mathrm{v})$ over night to hydrolyze the acetyl groups. After deacetylation the cellulose nanofibers mats were washed thoroughly with deionized water to remove sodium and acetate ions.

Pretreatment

Two methods for introducing carboxyl groups on the cellulose nanofiber surface were used. Cellulose nanofiber mats were treated with $5 \% \mathrm{w} / \mathrm{v} \mathrm{NaOH}$ solution and sodium chloroacetate $\left(1 \mathrm{~mol} \mathrm{dm}^{-3}\right)$ for $2 \mathrm{~h}$. Thereafter the mats were washed several times with deionized water and finally with ethanol.

Carboxymethyl cellulose, CMC, was adsorbed on cellulose nanofiber mats using a method previously published by others [21]. Cellulose nanofiber mats were immersed in an aqueous solution containing sodium carboxymethyl cellulose $\left(250 \mathrm{mg} \mathrm{dm}{ }^{-3}\right)$ and $\mathrm{CaCl}_{2}\left(0.01 \mathrm{~mol} \mathrm{dm}^{-}\right.$ $\left.{ }^{3}\right)$ for $24 \mathrm{~h}$. The nanofibers samples were then immersed a $\mathrm{CaCl}_{2}$ solution $\left(0.1 \mathrm{~mol} \mathrm{dm}^{-3}\right)$ for 24 $\mathrm{h}$ to introduce calcium counter ions to the surface. The nanofiber mats were then washed thoroughly with deionized water. The role of $\mathrm{CaCl}_{2}$ was to enhance the irreversible adsorption of CMC on to the cellulose surface [17].

Synthesis of HKUST-1 using layer-by-layer method

The nanofiber mats were immersed in copper acetate solution $\left(1 \mathrm{mmol} \mathrm{dm}^{-3}\right)$ of ethanol: Dimethylformamide (DMF) (1:1 v/v) for 15 minutes and then washed with ethanol:DMF. After washing, the mats were immersed in 1,3,5-benzenetricarboxylic acid (BTC) (1 mmol $\mathrm{dm}^{-3}$ ) for 30 minutes and then washed again with ethanol:DMF solvent. These four steps formed one cycle. The ethanol:DMF solution was changed into fresh solution after every fifth cycle and metal salt/ligand solutions were freshly prepared after every eight cycles. The 
This is an accepted manuscript, The final version can be found in Advanced Engineering Materials DOI:10.1002/adem.201400565

nanofiber mats were washed ethanol:DMF solution after synthesis and dried in ambient condition.

\section{Characterization}

FTIR spectrums were collected with a Perkin Elmer 2000 FT-IR using an attenuated total reflection module, which enabled sample surfaces to be examined without further preparation. Thermogravimetric Analysis (TGA) was preformed with a Perkin Elmer TGA 7 using $\mathrm{N}_{2}$ as purge gas. Samples were dried at $160{ }^{\circ} \mathrm{C}$ before analysis and the temperature gradient was 5 ${ }^{\circ} \mathrm{C} / \mathrm{min}$.

Micro structural investigations were performed using Scanning Electron Microscopy (SEM) with a LEO Ultra 55 FEG SEM. The SEM was operated at an acceleration voltage of $1.5 \mathrm{kV}$ and all samples were gold sputtered in a vacuum for 80 seconds at $10 \mathrm{~mA}$.

Powder X-ray diffraction patterns were recorded on a Siemens Diffractometer D5000 using vertical goniometer in Bragg-Brentano geometry and Cu-radiation of $45 \mathrm{kV}$ and $40 \mathrm{~mA}$.

$\mathrm{N}_{2}$ adsorption data was measured from 0-1 bar at $77 \mathrm{~K}$ on a Quantachrome instruments Autosorb-1c instrument at the University of Nottingham under ultra high vacuum in a clean system with a diaphragm and turbo pumping system. Samples were activated before analysis by heating at $150{ }^{\circ} \mathrm{C}$ for $20 \mathrm{~h}$. The multipoint BET surface areas were calculated by using the software (version 1.61) integrated in the instrumentation.

Received: ((will be filled in by the editorial staff))

Revised: ((will be filled in by the editorial staff)) Published online: ((will be filled in by the editorial staff))

_[1] a) L. Öhrström, K. Larsson Molecule-Based Materials: The Structural Network Approach, Elsevier, Amsterdam 2005; b) S.R. Batten, N.R. Champness, X.-M. Chen, J. Garcia-Martinez, S. Kitagawa, L. Öhrström, M. O’Keeffe, M.P. Suh, J. Reedijk, Pure Appl. Chem. 2013, 85, 1715; c) A. Bétard and R.A. Fischer, Chem. Rev. 2012, 112, 1055; d) Y. Cui, Y. Yue, G. Qian, and B. Chen, Chem. Rev. 2012, 112, 1126; e) L.E. Kreno, K. Leong, O.K. Farha, M. Allendorf, R.P. Van Duyne, and J.T. Hupp, Chem. Rev. 2012, 112, 1105. 
This is an accepted manuscript, The final version can be found in Advanced Engineering Materials DOI:10.1002/adem.201400565

_[2] a) U. Mueller, M. Schubert, F. Teich, H. Puetter, K. Schierle-Arndt, and J. Pastré, J. Mater. Chem. 2006, 16, 626; b) A. Corma, H. García, and F.X. Llabrés I Xamena, Chem. Rev. 2010, 110, 4606; c) Y. Liu, W. Xuan, and Y. Cui, Adv. Mater. 2010, 22, 4112; d) A.U. Czaja, N. Trukhan, and U. Müller, Chem. Soc. Rev. 2009, 38, 1284; e) G. Férey, C. Serre, T. Devic, G. Maurin, H. Jobic, P.L. Llewellyn, G. DeWeireld, A. Vimont, M. Daturi, and J.-S. Chang, Chem. Soc. Rev. 2011, 40, 550.

_ [3] D. Zacher, O. Shekhah, C. Wöll, and R.A. Fischer, Chem. Soc. Rev. 2009, 38, 1418.

_ [4] O. Shekhah, H. Wang, S. Kowarik, F. Schreiber, M. Paulus, M. Tolan, C. Sternemann, F. Evers, D. Zacher, R.A. Fischer, and C. Wöll, J. Am. Chem. Soc. 2007, 129, 15118.

_ [5] N. Stock and S. Biswas, Chem. Rev. 2012, 112, 933.

_ [6] H. Gliemann and C. Wöll, Mater. Today 2012, 15, 110.

_ [7] a) A.R. Abbasi, K. Akhbari, and A. Morsali, Ultrason. Sonochem. 2012, 19, 846; b) S. Khanjani and A. Morsali, Ultrason. Sonochem. 2014, 21, 1424; c) M. da Silva Pinto, C.A. Sierra-Avila, and J.P. Hinestroza, Cellulose 2012, 19, 1771; d) P. Küsgens, S. Siegle, and S. Kaskel, Adv. Eng. Mater. 2009, 11, 93.

_ [8] R. Ostermann, J. Cravillon, C. Weidmann, M. Wiebcke, and B.M. Smarsly, Chem. Commun. 2011, 47, 442.

_ [9] M. Rose, B. Böhringer, M. Jolly, R. Fischer, and S. Kaskel, Adv. Eng. Mater. 2011, 13,356 .

_ [10] Y.-N.Wu, F. Li, H. Liu,W. Zhu, M. Teng, Y. Jiang,W. Li, D. Xu, D. He, P. Hannam, and G. Li, J. Mater. Chem. 2012, 22, 16971.

_ [11] A. Centrone, Y. Yang, S. Speakman, L. Bromberg, G. C. Rutledge, and T. A. Hatton, J. Am. Chem. Soc. 2010, 132,15687.

_ [12] R. Jin, Z. Bian, J. Li, M. Ding, and L. Gao, Dalton Trans. 2013, 42, 3936.

_ [13] Z. Lian, L. Huimin, and O. Zhaofei, Dalton Trans. 2014, 43, 6684.

_ [14] Klemm, D., Heublein, B., Fink, H.-P. and Bohn, A, Angew. Chem. 2005, 117, 3422; Angew. Chem. Int. Ed. 2005, 44, 3358.

_ [15] a) S. Xu, J. Zhang, A. He, J. Li, H. Zhang, C. C. Han, Polymer 2008, 49, 2911; b) M. G. Freire, A. R. Teles, R. A. S. Ferreira, L. D. Carlos, J. A. Lopes-da-Silva, J. A. P. Coutinho, Green Chem. 2011,13, 3173; c) L. Härdelin, J. Thunberg, E. Perzon, G. Westman, P.

Walkenström, and P. Gatenholm, J. Appl. Polym. Sci. 2012, 125, 1901.

_ [16] H. Liu and Y.-L. Hsieh, J. Polym. Sci. B Polym. Phys. 2002, 40, 2119.

_ [17] J. Laine, T. Lindström, G.G. Nordmark, and G. Risinger, Nord. Pulp Pap. Res. J. 2000, 15, 520 .

_ [18] S. S.-Y. Chui, S. M.-F. Lo, J. P. H. Charmant, A. G. Orpen, and I. D. Williams, Science 1999, 283, 1148.

_[19] a) A.A. Yakovenko, J.H. Reibenspies, N. Bhuvanesh, and H.-C. Zhou, J. Appl.

Crystallogr. 2013, 46, 346; b) F.H. Allen, Acta Crystallogr., Sect. B: Struct. Sci. 2002, 58, 380.

_ [20] Y. Li and R.T. Yang, AIChE J. 2008, 54, 269.

_ [21] K. Rodríguez, S. Renneckar, and P. Gatenholm, ACS Appl. Mater. Interfaces 2011, 3, 681. 
This is an accepted manuscript, The final version can be found in Advanced Engineering Materials DOI:10.1002/adem.201400565

J. Thunberg*, E. Laurila, S. P. Argent, N. R. Champness, S. Zacharias, G. Westman* and L. Öhrström

$\mathbf{\square}<\mathrm{B}>\ldots<\mathrm{B}>$

Carboxyl anchor points enchances Metal organic framework (MOF) formation and distribution on the nanofibers surface. Two methods for introducing carboxyl anchor points on the surface of electrospun cellulose are evaluated. The SEM image (top) show uneven crystal growth on untreated plain cellulose nanofibers. MOF crystals are visible covering the whole nanofiber surface of pretreated electrospun cellulose (bottom). Synthesis of MOF on the surface greatly increase the specific surface area of the nanofiber material.

\section{ToC figure ((48 $\mathrm{mm}$ broad $))$}
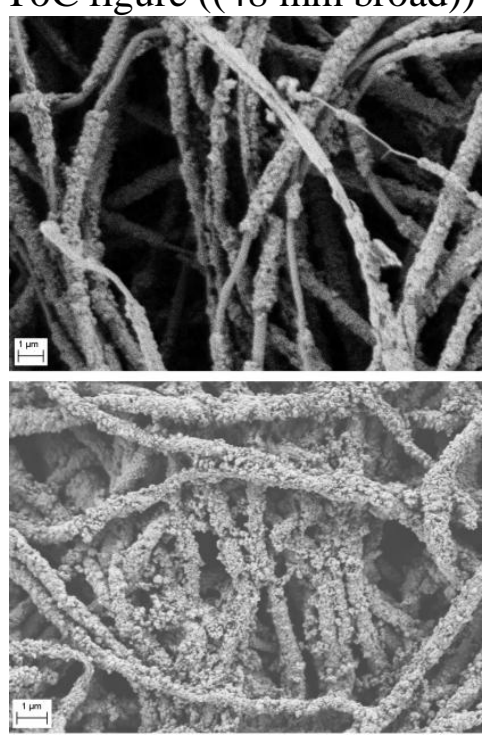